\title{
InGaAs/InP hot electron transistors grown by chemical beam epitaxy
}

\author{
W. L. Chen, J. P. Sun, G. I. Haddad, M. E. Sherwin, G. O. Munns, J. R. East, \\ and R. K. Mains \\ Center for High Frequency Microelectronics, Department of Electrical Engineering and Computer \\ Science, The University of Michigan, Ann Arbor, Michigan 48109-2122
}

(Received 3 February 1992; accepted for publication 23 April 1992)

In this letter, we report on the dc performance of chemical beam epitaxy grown InGaAs/InP hot electron transistors (HETs). The highest observed differential $\beta\left(d I_{C} / d I_{B}\right)$ is over 100 . The HETs have Pd/Ge/Ti/Al shallow ohmic base contacts with diffusion lengths less than $300 \AA$. Furthermore, we also demonstrated ballistic transport of electrons in an InGaAs/InP HET by obtaining an energy distribution of electrons with $\sim 60 \mathrm{meV}$ full width at half maximum. The measured conduction band discontinuity of InGaAs/InP is $250.3 \mathrm{meV}$, which is $39.8 \%$ of the band gap difference.

Hot electron transistors (HETs) have been studied for high speed applications during the last decade, starting from GaAs/AlGaAs HETs. ${ }^{1}$ Heiblum et al. ${ }^{2}$ used the same material system and reported a differential $\alpha\left(d I_{C} / d I_{E}\right)$ $=0.9$. In order to obtain an even higher $\alpha$, a pseudomorphic InGaAs base device, which offered larger $\Gamma$ to $L$ valley separation, was introduced by Seo et al. ${ }^{3}$ They observed a differential $\beta\left(d I_{C} / d I_{B}\right)$ as high as 41 at $4.2 \mathrm{~K}$. Because InGaAs and InP have wider $\Gamma$ to $L$ valley separation and higher electron saturation velocity than the GaAs/AlGaAs system, the InGaAs/InP $\mathrm{HET}^{4}$ was predicted to have higher current gain and better switching speed.

The HET structure is difficult to fabricate because of the problems in making an ohmic contact to a narrow base region. In previous work, $\mathrm{Cr} / \mathrm{Au}^{5}$ and $\mathrm{Au} / \mathrm{Ge} / \mathrm{Ni}^{6}$ systems were the most-used metals for nonalloyed base contacts. However, the base had to be heavily doped, which limited the gain of the HETs. Furthermore, the nonalloyed $\mathrm{Cr} / \mathrm{Au}$ or $\mathrm{Au} / \mathrm{Ni} / \mathrm{Ge}$ contact has a high contact resistance at low temperature if the base is not heavily doped and this degrades the high frequency performance. In this study, we used a $\mathrm{Pd} / \mathrm{Ge} / \mathrm{Ti} / \mathrm{Al}$ ohmic system to contact a thin base layer $(400 \AA) .^{7}$ This ohmic system has a shallow diffusion length $(<300 \AA$ ) and shows good ohmic behavior at $80 \mathrm{~K}$.

In this work, we used the InGaAs/InP material system to study the dc performance of the HETs. Moreover, an "energy spectrometer" 1,2 technique was used to investigate the ballistic transport of electrons and measure the collector barrier height of the HETs.

The materials of this study were grown using a Varian Gen II chemical beam epitaxy (CBE) reactor. The source materials were trimethylindium, triethylgallium, $100 \%$ phosphine and $100 \%$ arsine for $\mathrm{In}_{0.53} \mathrm{Gr}_{0.47}$ As and InP. All the structures were grown on (100) Fe-doped semiinsulating InP substrates at a substrate temperature of $530^{\circ} \mathrm{C}$. The typical HET structure is shown in Fig. 1 .

The fabrication of the HETs was conducted by a novel self-aligned process. First, conventional photolithography was used for all of the patternings. Second, we adopted $\mathrm{HBr} / \mathrm{H}_{2} \mathrm{O} / \mathrm{SWBr}$ (saturated bromine water) for nonselective mesa etching, and $\mathrm{H}_{3} \mathrm{PO}_{4} / \mathrm{H}_{2} \mathrm{O}_{2} / \mathrm{H}_{2} \mathrm{O}$ for selective base recess. Then the $\mathrm{Ni} / \mathrm{Ge} / \mathrm{Au} / \mathrm{Ti} / \mathrm{Au}$ and $\mathrm{Pd} / \mathrm{Ge} / \mathrm{Ti} /$ Al system were used for the emitter (collector) and base contacts. After the fabrication of the devices, we conducted all dc measurements of the HETs in a cryogenic cooling stage using a HP4145B system at $80 \mathrm{~K}$.

When the HET is operated under forward bias mode (as shown in Fig. 2), e.g., common base configuration, small emitter current will modulate the voltage across the emitter barrier, resulting in the modulation of collector current. For the HET with a base width $\left(W_{b}\right)=400 \AA$ and a base doping $\left(N_{d}\right)=1 \times 10^{17} \mathrm{~cm}^{-3}$, the highest differential $\alpha$ observed was over 0.99 , which corresponded to a differential $\beta$ over 100 . The common base $I-V$ characteristics of this HET are shown in Fig. 3.

We studied the dc performances of the HETs by changing the base width $\left(W_{b}\right)$ and base doping $\left(N_{d}\right)$. We found that as $W_{b}$ and $N_{d}$ were decreased, the probability of scattering was decreased, thus the differential $\alpha$ was increased. However, when $N_{d}$ is increased, the output conductance of the HET is decreased, and the surface depletion depth is also decreased at the extrinsic base region, although the differential $\beta$ is decreased. Thus, we could obtain better $I-V$ characteristics from the HET. For $N_{d}=2$ $\times 10^{17} \mathrm{~cm}^{-3}, W_{b}=400 \AA$ (UMA-580), the common emitter $I-V$ characteristics are shown in Fig. 4.

As shown in Fig. 4, for every $I_{B}$ step, $I_{C}$ dramatically increases roughly at $V_{C E}=1.0 \mathrm{~V}$. This could be the combined effects of (1) tunneling of base electrons through the

\begin{tabular}{lccl} 
layer & doping $\left(\mathrm{c} \mathrm{m}^{-3}\right)$ & width $(\mathrm{n} \mathrm{m})$ \\
\hline InGaAs & $1.0 \times 10^{18}$ & 300 & Emitter \\
\hline InGaAs & undoped & 5 & Spacer \\
\hline InP & undoped & 17.5 & Emitter barrier \\
\hline InGaAs & undoped & 5 & Spacer \\
\hline InGaAs & Nd & Wb - 10 & Base \\
\hline InGaAs & undoped & 5 & Spacer \\
\hline InP & undoped & 200 & Collector barrier \\
\hline InGaAs & undoped & 5 & Spacer \\
\hline InGaAs & $1.0 \times 10^{18}$ & 400 & Collector \\
\hline \multicolumn{5}{c}{ InP } & buffer & layer \\
\hline
\end{tabular}

Fe-doped Semi-insulating InP substrate

FIG. 1. The HET structure with base width $W_{b}$ and base doping $N_{d^{*}}$ 


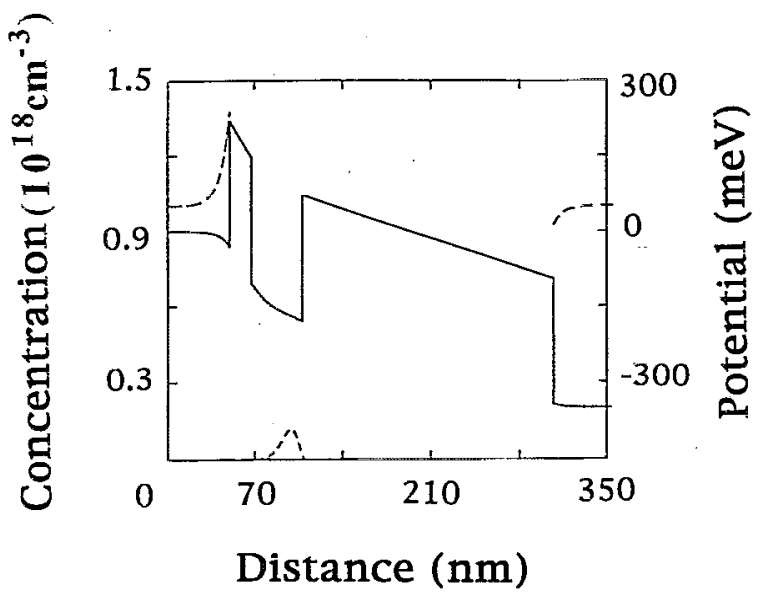

FIG. 2. The band diagram of the HET under forward bias mode, which is generated by a self-consistent simulation, solid line=conduction band, dashed line $=$ electron concentration.

collector barrier and (2) depletion of base charge (punch through). Moreover, the HET has an $I_{C}$ turn-on voltage $V_{C E} \sim 0.2 \mathrm{~V}$. Below this voltage, the emitter and collector barriers block low energy electrons efficiently, resulting in very small $I_{C}$ output current. The differential $\beta$ is from 32 to $\mathbf{4 5}$ for most of the bias range, and the highest differential $\beta$ is 66. Moreover, we found that further increasing of $N_{d}$ $\left(>3 \times 10^{17} \mathrm{~cm}^{-3}\right)$ and $W_{b}$ resulted in a decrease of differential $\beta$ and $\alpha$.

One of the advantages of studying HETs is to provide information about ballistic transport of electrons in semiconductors. Previous researchers have demonstrated this phenomenon in GaAs/AlGaAs, ${ }^{1}$ InGaAs/AlGaAs, ${ }^{2}$ and InAlAs/InGaAs ${ }^{8}$ HETs. In this study, we demonstrated this phenomenon in an InGaAs/InP HET for the first time with the UMA-555 HET, using an energy spectrometer technique.

For the HET operating in the energy spectrometer mode, which implies that both $V_{E B}$ and $V_{C B}$ are negative, the energy band diagram is shown in Fig. 5 . The derivative of $I_{C}$ with respect to $V_{C B}$, i.e., $d I_{C} / d V_{C B}$ is proportional to

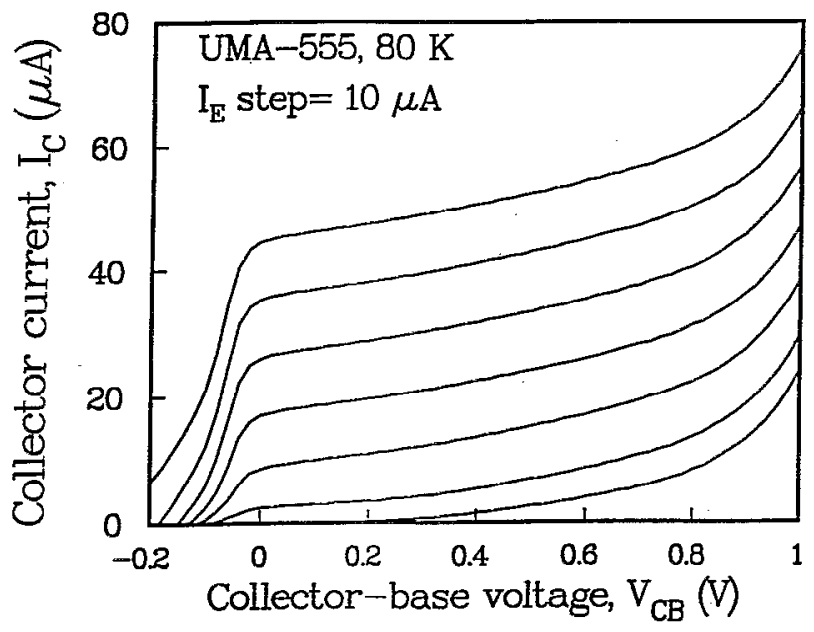

FIG. 3. Common base $I-V$ characteristics of the UMA-555 HET.

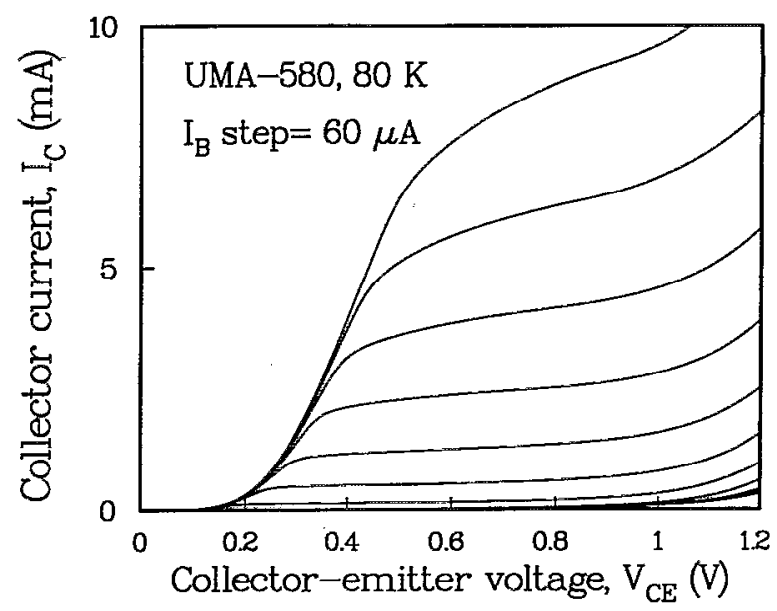

FIG. 4. Common emitter $I-V$ characteristics of the UMA-580 HET.

the number of collected electrons as a function of energy. By varying $V_{C B}$, we will get a distribution of the collected electrons, as shown in Fig. 5. The main peak of this distribution is the ballistic peak ${ }^{2}$ and the peak value is at $V_{C B}$ $=V_{p}$. The full width at half-maximum (FWHM) of the distribution remains around $60 \mathrm{meV}$ for different $V_{E B}$.

As discussed in Refs. 2 and 8, we can estimate the collector barrier height $\Phi_{C}$ from the ballistic condition, as

$$
q V_{E B}-\Delta=\Phi_{C}+q V_{C B} \text { (at peaks) } \cdots \delta_{C}-\zeta \text {. }
$$

Basically, this condition implies the energy conservation of ballistic electrons for the entire transpurt. Where $V_{E B}$ is the injection voltage; $\Delta$ is the small displacement of the normal distribution peak injected into the base down from the Fermi level in the emitter; $\Phi_{C}$ is the collector barrier height; $V_{C B}$ (at peak) is equal to $V_{p} ; \delta_{c}$ is the band bending at the collector side of the spectrometer barrier at $V_{C B}$ $=V_{p}$; and $\zeta=E_{F}-E_{C}$ is assumed to be $82.5 \mathrm{meV}$ for $n_{c}=1$ $\times 10^{18} \mathrm{~cm}^{-3}$ at $80 \mathrm{~K}$.

In Eq. (1), the $V_{E B}$ and $V_{C B}$ are measured values, and the $\Delta$ and $\delta_{c}$ are calculated by a HET simulation with an

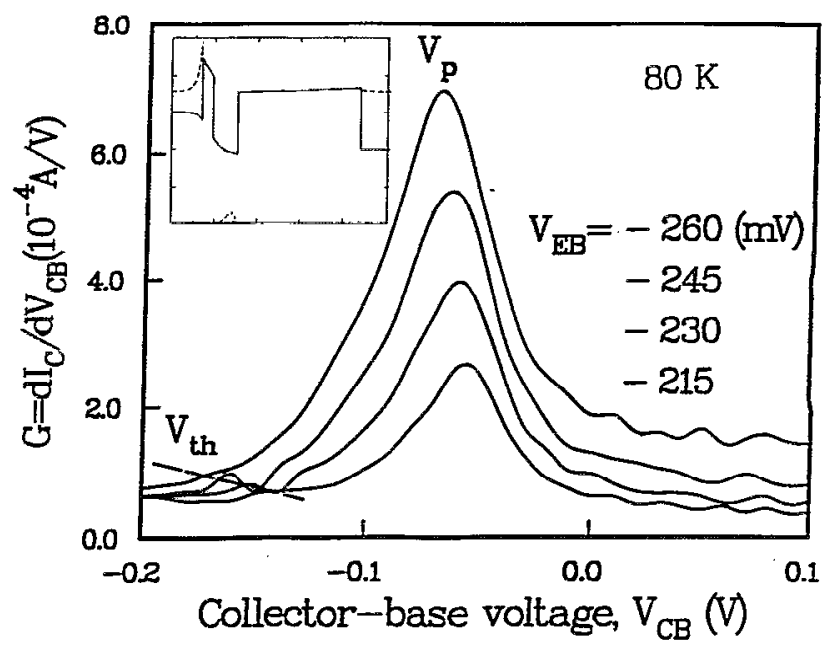

FIG. 5. $G$ vs $V_{C B}$ curve; and the band diagram under the energy spectrometer mode, solid line $=$ conduction band, dashed line $=$ electron concentration. 
TABLE I. Summary of energy distribution results.

\begin{tabular}{lcclll}
\hline \hline $\begin{array}{l}-V_{E B} \\
(\mathrm{mV})\end{array}$ & $\begin{array}{c}\Delta \\
(\mathrm{meV})\end{array}$ & $\begin{array}{c}\delta_{C} \\
(\mathrm{meV})\end{array}$ & $\begin{array}{c}-V_{p} \\
(\mathrm{mV})\end{array}$ & $\begin{array}{c}\Phi_{C} \\
(\mathrm{meV})\end{array}$ & $\begin{array}{c}-V_{\text {th }} \\
(\mathrm{mV})\end{array}$ \\
\hline 215 & 11.0 & 0.5 & 50 & 237 & 142.5 \\
230 & 10.0 & 0.8 & 57.5 & 245.8 & 150 \\
245 & 8.9 & 1.0 & 65 & 254.6 & 160 \\
260 & 7.5 & 1.5 & 72.5 & 264 & 172.5 \\
\hline
\end{tabular}

assumed barrier height of $250 \mathrm{meV}$. These values are listed in Table $I$ for various bias conditions. The $\Delta$ becomes smaller as $V_{E B}$ increases to more negative values, since emitter electrons see a thinner triangular emitter barrier, resulting in less reflection of electrons and a smaller $\Delta$. From Eq. (1), the calculated collector barrier height ranges from 237 to $264 \mathrm{meV}$ with an average of $250.3 \mathrm{meV}$. It agrees well with the number assumed in the HET simulation $(250 \mathrm{meV})$. Moreover, the Fermi level is assumed almost at the conduction band edge in the base region. Therefore, the above result suggests that the conduction band discontinuity is $39.8 \%$ of the band gap difference for the InP/InGaAs heterojunction, and it is consistent with previous reports ${ }^{9,10}$ which obtained the data from admittance spectroscopy or $C-V$ measurements.

The FWHM of the distribution is not broadened for different $V_{E B}$ and the peak value satisfies the ballistic condition [Eq. (1)] within the energy associated with an optical phonon scattering ( $34.5 \mathrm{meV}$ for InGaAs). Moreover, for the $V_{E B}$ 's shown in Fig. 5, we measured the base transport ratio $\alpha\left(I_{C} / I_{E}\right)$ at $V_{C B}=V_{p}$ to determine the fraction of ballistic electrons. From the two conditions and measured $\alpha$ 's, we found that up to $50 \%$ of emitter electrons could travel through the base region ballistically.

Furthermore, it is shown in Fig. 5 that the $G\left(d I_{C}\right)$ $d V_{C B}$ ) value does not drop to zero within the voltage range shown in Fig. 5; e.g., we find $I_{C}$ drops below zero at a threshold voltage $V_{\mathrm{th}}$. This is because, when $V_{C B}<V_{\mathrm{th}}$, electrons start to tunnel through the collector barrier from the collector to the base, resulting in negative $I_{C}$. If $I_{C}$ remains unchanged for a range of voltage, $d I_{C} / d V_{C B}$ will drop to zero. However, we did not observe such a range of voltage in our HET structure.

In conclusion, we have fabricated CBE grown InGaAs/InP HETs with high current gains and shallow ohmic base contacts. The processing technique developed in this study can be used to fabricate other types of resonant tunneling transistors with direct contact to the base. The InP/InGaAs material system is promising for high speed device applications. Moreover, the HET is a good tool to investigate carrier transport in semiconductors and physical properties of semiconductors.

This work was supported by the Army Research Office under the URI program Contract No. DAAL03-87-K0007.

${ }^{1}$ N. Yokoyama, K. Imamura, T. Ohshima, N. Nishi, S. Muto, K. Kondo, and S. Hiyammzu, IEEE Digest IEDM 532 (1984).

${ }^{2}$ M. Heiblum and M. V. Fischetti, in Physics of Quantum Electron Devices, edited by F. Capasso (Springer, Berlin, 1990), Chap. 9.

${ }^{3}$ K. Seo, M. Heiblum, C. M. Knoedler, J. E. Oh, J. Pamulapati, and P. Bhattacharya, IEEE Electron Device Lett. EDL-10, 73 (1989).

${ }^{4}$ S. Yamaura, Y. Miyamoto, and K. Furuya, Electron. Lett. 26, 1055 (1990).

${ }^{5}$ K. Uesaka, S. Yamaura, Y. Miyamoto, and K. Furuya, Electron. Lett. 25, 705 (1989).

${ }^{6}$ I. Hase, K. Taira, H. Kawai, T. Watanabe, K. Kaneko, and N. Watanabe, Elcctron. Lett. 24, 279 (1988).

${ }^{7}$ W. L. Chen, J. P. Sun, G. I. Haddad, M. E. Sherwin, G. O. Munns, J. R. East, and R. K. Mains, Proceedings of the 1991 International Semiconductor Devices Research Symposium, Charlottesville, VA, p. 297.

${ }^{8}$ U. K. Reddy, J. Chen, C. K. Peng, and H. Morkoc, Appl. Phys. Lett. 48, 1799 (1986).

${ }^{9}$ D. V. Lang, M. B. Panish, F. Capasso, J. Allam, R. A. Hamm, and A. M. Sergent, J. Vac. Sci. Technol. B 5, 1215 (1987).

${ }^{10}$ M. T. Furtado, M. S. S. Loural, A. C. Sachs, and P. J. Shieh, Superlatt. Microstruct. 5, 507 (1989). 